\title{
Exclusion of a primary gene defect at the HLA locus in familial idiopathic dilated cardiomyopathy
}

\author{
T M Olson, S N Thibodeau, P A Lundquist, D J Schaid, V V Michels
}

\begin{abstract}
Case control studies have reported associations between specific HLA class II antigens and idiopathic dilated cardiomyopathy (DCM), suggesting that genetically regulated immune response factors may be involved in the pathogenesis of this disease. In this study, families with DCM were used to test the hypothesis that a heritable gene defect in the HLA region is the primary genetic determinant for a subset of cases. Twelve families with DCM were identified. By formal segregation analysis, the inheritance of the disease was most consistent with an autosomal dominant gene defect with incomplete penetrance. Genotyping was performed with five highly polymorphic linked dinucleotide repeat markers that span the HLA locus. Linkage analysis was used to determine whether or not these genetic markers cosegregated with the disease phenotype. Genetic linkage between the disease phenotype and a $21 \mathrm{cM}$ region spanning the HLA was excluded (lod score $\leq-2$ ) in at least $60 \%$ of our families. These results indicate that a gene defect in the HLA locus region is not the primary genetic determinant of DCM in a series of familial cases. However, our data do not exclude the possibility that HLA regulated immune response factors may have a modifying effect on disease penetrance and expression.
\end{abstract}

( $\mathcal{F}$ Med Genet 1995;32:876-880)

Departments of Laboratory Medicine and Pathology, Medical Genetics, Biostatistics, and Section of Pediatric Cardiology, Mayo Clinicl

Foundation,

Rochester, MN 55905,

USA

$\mathrm{T} M$ Olson

S N Thibodeau

P A Lundquist

D J Schaid

V V Michels

Correspondence to: Dr Michels, Department of Medical Genetics,

Mayo Clinic, Rochester, MN 55905, USA.

Received 11 January 1995 Revised version accepted for

publication

26 June 1995
Dilated cardiomyopathy, characterised by ventricular enlargement and dysfunction, is a final common pathway for multiple disease processes. By definition, idiopathic dilated cardiomyopathy (DCM) excludes identifiable systemic diseases, coronary artery disease, myocarditis, and toxic insults leading to myocardial failure. Nevertheless, DCM is thought to be a heterogeneous disorder. Case-control studies have implicated immunological, ${ }^{12}$ occult infectious, ${ }^{3}$ and biochemical ${ }^{4}$ factors in the disease pathogenesis. Approximately 20 to $25 \%$ of patients with DCM have familial disease but the specific genetic aetiology is unknown in the majority of families.

The potential role of immune mechanisms in myocardial disease has been reviewed recently. ${ }^{5}$ It has been hypothesised from HLA typing studies that there may be a subset of DCM patients in whom immune response factors may play a role in the disease pathogenesis. Previous studies have shown a correlation between specific HLA class II antigens, as assigned by cytotoxicity assay, and DCM. ${ }^{1}$ However, the association is not universal.

A meta-analysis of five studies showed a statistically significant increased frequency of HLA-DR4 in cases of DCM (odds ratio 2.06) and also suggested an additional association with DQw4. ${ }^{1}$ HLA typing studies are complicated by potentially misleading conclusions inherent in traditional serological assays ${ }^{6}$ and difficulties in ascertaining a true control group owing to ethnic and geographical variation of HLA antigens. ${ }^{7}$ When association studies for DCM with class II major histocompatibility studies were performed using DNA genomic typing, a weaker correlation was found for DR alleles that was not statistically significant. Furthermore, DR4 subtypes were dispersed equally among patients with DCM. ${ }^{8}$ The authors concluded that their data did not support the hypothesis that DCM results from a single leucocyte antigen complex, and raised the possibility of other, more complex immune mechanisms. ${ }^{8}$ It was hypothesised that genes linked to HLA alleles might contribute to the aetiology of DCM.

Case-control association studies do not allow the distinction between a disease modifying gene and a primary gene defect that is fundamental to the development of disease. Linkage studies of familial DCM allow for detection of such a primary genetic aetiology in the HLA region. Linkage studies of familial DCM also would show the presence of a non-HLA allele within this chromosomal region that might result in DCM and that could explain the reported HLA associations, which generally have been weak. Few investigations of the HLA locus in familial DCM have been reported. ${ }^{2910}$

Therefore, we evaluated 12 families with $\mathrm{DCM}^{1112}$ by linkage analysis to test the hypothesis that DCM in these family members was caused primarily by an HLA regulated defect in immune response or by some other unidentified gene in the HLA region. By formal segregation analysis, these families as a group fit a model of inheritance that was $10^{10}$ times more likely than other single genes or multifactorial models. 

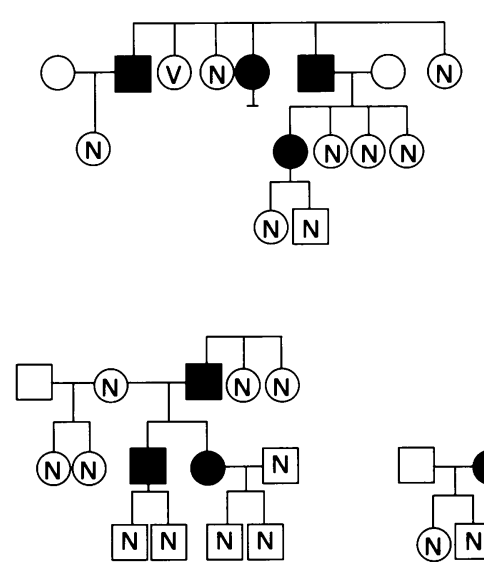

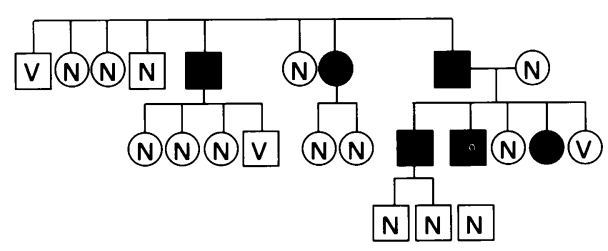

(N)

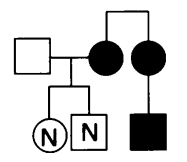

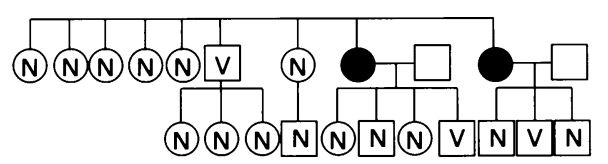
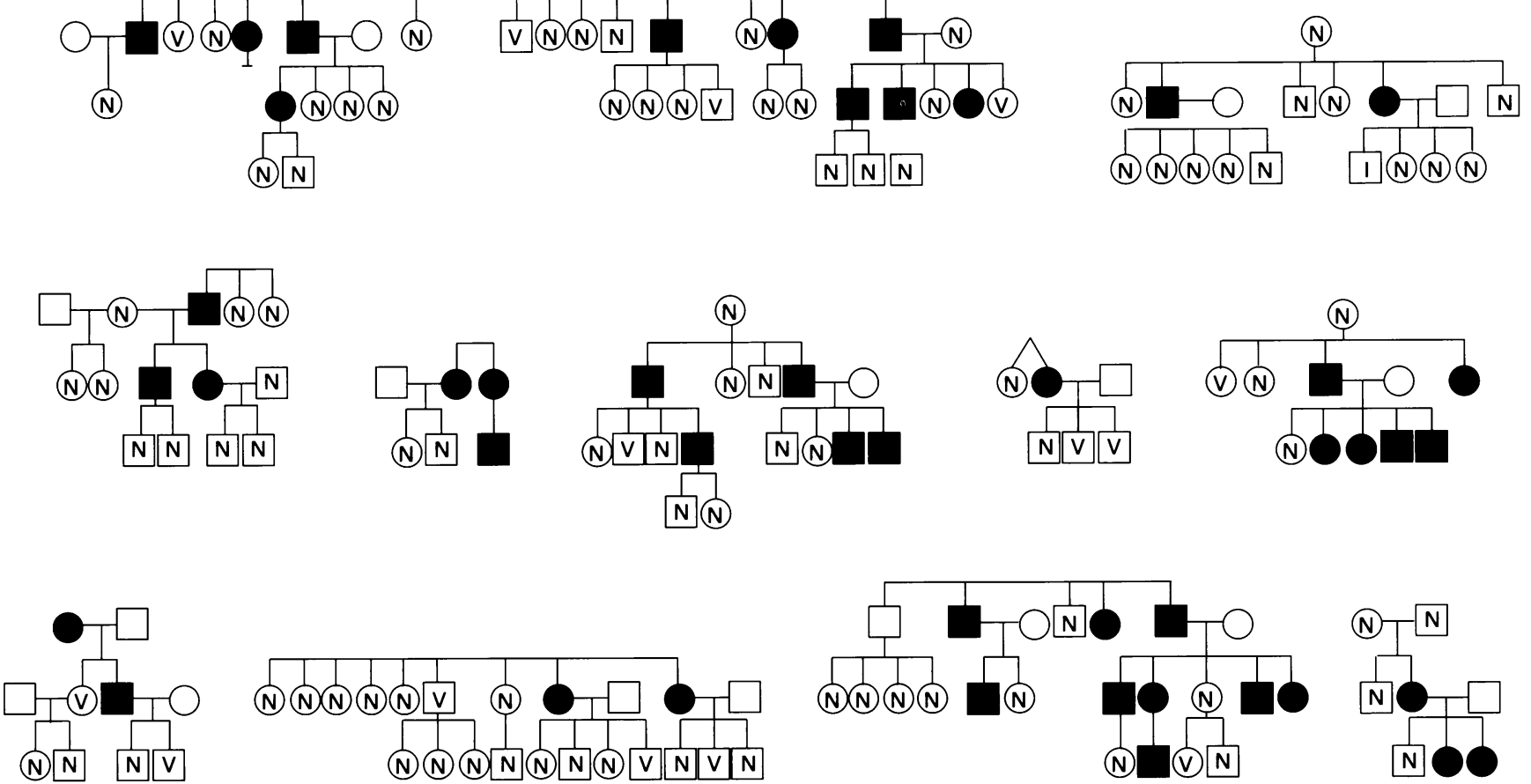

Figure 1 Pedigrees of DCM families. Only subjects for whom DNA was available are shown in these pedigrees. Shaded symbols represent affected subjects. " $N$ " in symbol represents those who were classified as unaffected after comprehensive evaluations. "V" in symbol represents persons with a slightly enlarged left ventricle but normal ejection fraction.

\section{Materials and methods PATIENTS}

Familial cases of DCM were identified as previously described. ${ }^{112}$ Diagnostic criteria included a left ventricular ejection fraction $<50 \%$ by echocardiography or cardiac catheterisation, a left ventricular diastolic dimension $>95$ th centile for age and body surface area, and documentation by coronary angiography of no coronary artery segment with $\geq 50 \%$ obstruction in patients older than 40 years. Patients with coronary artery disease $(\geq 50 \%$ occlusion of any segment), congenital malformations, intrinsic valve disease, systemic diseases, or myocarditis were excluded. The study was approved by the institutional review board and participants gave informed, written consent.

First degree relatives of the index patients were evaluated by physical examination, electrocardiography, and comprehensive two dimensional and $M$ mode echocardiography. Twelve DCM families with up to nine affected members (fig 1) were identified for molecular genetic studies. In some families, members with ventricular enlargement, but a left ventricular ejection fraction $\geq 50 \%$, were identified and classified as uncertain phenotype. Formal segregation analysis determined that the disease inheritance in these families, as a group, was most consistent with an autosomal dominant locus with incomplete penetrance, ${ }^{11}$ even though in three of 12 DCM pedigrees, parents were not available for study.

\section{DNA ANALYSIS}

Approximately $20 \mathrm{ml}$ of whole blood was collected from affected and unaffected family members. A buffy coat was prepared from one portion of the sample and DNA was isolated using an automated extraction procedure (Applied Biosystems Inc, model 340A), according to the manufacturer's instructions. The remaining portion was used to establish an EBV transformed $B$ cell line by a modification of the method of Roder et al. ${ }^{13}$ DNA was extracted from paraffin embedded tissue blocks from two affected subjects who had died.

Because the entire HLA locus is $>3500$ kilobases $^{7}$ and a single marker may not be informative for linkage in all families, five linked markers mapped to the chromosome $6 \mathrm{p} 21.3$ band and spanning the HLA locus were used. These included flanking markers for the HLA region. All markers were highly polymorphic dinucleotide repeats of the $(\mathrm{CA})_{n}$ repeat type. ${ }^{14}$ The markers have been localised on published genetic maps ${ }^{15-17}$ and span a genetic distance of approximately $9 \mathrm{cM}$. D6S105 has been mapped $2 \mathrm{cM}$ telomeric to the HLA region ${ }^{15}$ and D6S291 has been mapped $4 \mathrm{cM}$ centromeric to the class II HLA-DRB1 locus of the HLA region. ${ }^{17}$ Oligonucleotide primers were synthesised by a core laboratory facility based upon published sequences ${ }^{1618}$ and diluted to a standard concentration of $20 \mu \mathrm{mol} / 1$.

Microsatellite markers were analysed by the polymerase chain reaction, using a modification of the method described by Weber and May. ${ }^{14}$ 
Two point lod scores between HLA marker loci and DCM at recombination fractions of zero, and regions of exclusion based on total lod scores

\begin{tabular}{lccccc}
\hline Pedigree & \multicolumn{5}{l}{ HLA marker loci, ordered telomeric to centromeric } \\
\cline { 2 - 6 } & D6S299 & D6S276 & D6S105 & D6S273 & D6S291 \\
\hline 1 & -1.12 & 0.45 & -4.21 & -5.95 & -4.38 \\
2 & -0.50 & -0.50 & 0.16 & -0.32 & -0.41 \\
3 & -2.82 & -2.09 & -3.58 & -0.81 & -0.38 \\
4 & -0.28 & -0.03 & -0.02 & -0.02 & -0.28 \\
5 & 0.80 & 0.80 & 0.25 & -0.32 & 0.80 \\
6 & 0.06 & 0.06 & -3.68 & 0.04 & -0.16 \\
7 & -0.76 & 0.00 & -0.71 & -0.74 & 0.00 \\
8 & 0.20 & -0.72 & -0.72 & 0.15 & 0.10 \\
9 & -0.48 & 0.14 & -0.74 & -0.94 & -0.81 \\
10 & 0.25 & -0.75 & -0.75 & -0.22 & -0.23 \\
11 & -0.72 & -0.43 & 0.14 & -0.73 & -0.72 \\
12 & -0.01 & 0.86 & -0.38 & -2.14 & -2.84 \\
Total & -5.37 & -2.22 & -14.24 & -11.98 & -9.31 \\
Exclusion & & & & & 9.0 \\
region (cM) & 5.3 & 0.3 & 18.5 & 18.6 & 9.5 \\
\hline
\end{tabular}

Each $10 \mu \mathrm{l}$ reaction consisted of $10 \mathrm{mmol} / 1 \mathrm{Tris}$, $1.5 \mathrm{mmol} / 1 \mathrm{MgCl}_{2}, 50 \mathrm{mmol} / \mathrm{KCl}, 200 \mu \mathrm{mol} /$ 1 each of dATP, dGTP, and dTTP, $40 \mu \mathrm{mol} / 1$ dCTP, $0.2 \mu \mathrm{mol} / 1$ of each primer, 0.5 units Taq polymerase, $1 \mu \mathrm{Ci} \alpha-{ }^{32} \mathrm{P}$ dCTP, and $8.6 \mu \mathrm{l}$ sterile water. Thermocycling (Perkin Elmer, GeneAmp PCR System, model 9600) was programmed for $94^{\circ} \mathrm{C} \times 30$ seconds, $55^{\circ} \mathrm{C} \times 30$ seconds, $72^{\circ} \mathrm{C} \times 30$ seconds for 30 cycles, then $72^{\circ} \mathrm{C} \times 10$ minutes. Samples were diluted 1:10 with four parts water and five parts loading buffer. Three $\mu \mathrm{l}$ was loaded and electrophoresed on a $6 \%$ polyacrylamide and $7 \mathrm{~mol} / \mathrm{l}$ urea gel at 70 watts for one to three hours. The gel was dried and bands were visualised by autoradiography after 2 to 24 hours exposure at room temperature.

\section{STATISTICAL ANALYSIS}

Two point linkage analysis ${ }^{19}$ was performed using the computer program LINKAGE version $5 \cdot 1 .{ }^{20}$ Alleles for the polymorphic loci were distinguished according to their relative size and segregation patterns, assuming equal allele frequencies. Other models assuming unequal allele frequencies had minimal effect on the lod scores. Because the age specific penetrance of DCM is unknown, we used two methods of analysis: (1) an age dependent penetrance with four age groups ( $\leq 20$ years with $5 \%$ penetrance, $21-30$ years with $20 \%$ penetrance, 31-40 years with $50 \%$ penetrance, and $>40$ years with $90 \%$ penetrance), and (2) affected only, by assigning unknown status to unaffected subjects so that they did not contribute to the lod score, but contributed only to determination of parental phase of the marker loci. Analyses were specifically formulated to decrease the possibility of falsely negative lod scores and, hence, false exclusion of the HLA locus owing to a misspecified analysis mode. Misspecification can be because of an underestimate of the disease allele frequency or of phenocopy rate, overestimate of penetrance, or failure to account for locus heterogeneity. Misspecification of the genetic model for the linkage analyses will not increase the chance of a false positive linkage finding, but can falsely inflate the region of exclusion. For these reasons, we used an inflated disease allele frequency of 0.05 for two point analysis as a robust method to absorb the misclassification of segregation, as suggested by Risch and $\mathrm{Gi}-$ uffra. ${ }^{21}$ In contrast, for multipoint analyses we used a more likely disease gene frequency of 0.0001 , but modelled locus heterogeneity directly by allowing for a proportion of linked families, $\alpha$, and a proportion not linked, $1-\alpha$. This is based on the admixture test in the HOMOG program. ${ }^{22}$ For multipoint exclusion, we estimated the smallest proportion, $\alpha$, that allowed exclusion of the HLA region as a candidate gene region. Subjects with left ventricular enlargement, but normal ejection fraction, were considered as unaffected in this report, because additional analyses treating them as affected did not substantially alter our reported results.

The genetic map of four of the five markers has been reported to be 299-(4 cM)-276(1 cM)-273-(4 cM)-291, where the distance in cM is given in brackets. ${ }^{16}$ Because D6S105 has not been mapped relative to each of the other four markers, multipoint linkage analysis was performed using the genotypes from the DCM families. The order for these five markers was 299-276-105-273-291 (maximum lod score of 35.31); further evidence for this order is given in Martin et al. ${ }^{23}$ The next highest peak lod score was 34.90 for the order of 299-276-273291-105. The former marker order was used for multipoint analyses. Both D6S273 and HLADRB1 have been localised $4 \mathrm{cM}$ centromeric to D6S291 on separate linkage maps, ${ }^{1617}$ indicating that, among the five markers, D6S273 is most closely linked to the HLA-DR region.

\section{Results}

Twelve DCM families were tested with each of the five markers spanning the HLA locus. Based on the age dependent penetrance model and two point analyses, all five markers spanning the HLA locus had lod scores $<-2$ at a recombination fraction of zero (table), effectively excluding the HLA locus as the primary gene defect in our families. There was no evidence of genetic heterogeneity for any of the five markers, with the smallest $p$ value of 0.17 for the marker D6S276, based on the admixture test using the HOMOG program. ${ }^{22}$

To combine the linkage information from the five markers, we performed multipoint linkage analyses. For this, only affected subjects were included. Multipoint analysis allowed exclusion of a $51 \mathrm{cM}$ region spanning the HLA region (fig 2). Again, there was no evidence of genetic heterogeneity using multipoint lod scores for the admixture test $(p=1 \cdot 0)$. However, because we had only 12 relatively small pedigrees, we had limited power to detect genetic heterogeneity. For this reason, we also performed multipoint exclusion allowing for a percentage of linked families. By decreasing the percentage of linked families from $100 \%$ (fig 2) to a lower value, we determined that we could exclude linkage to the HLA region when the percentage of linked families was as low as $60 \%$ (fig 3). That is, we could conservatively rule out the possibility that $60 \%$ or more of DCM is attributable to linkage with the HLA 


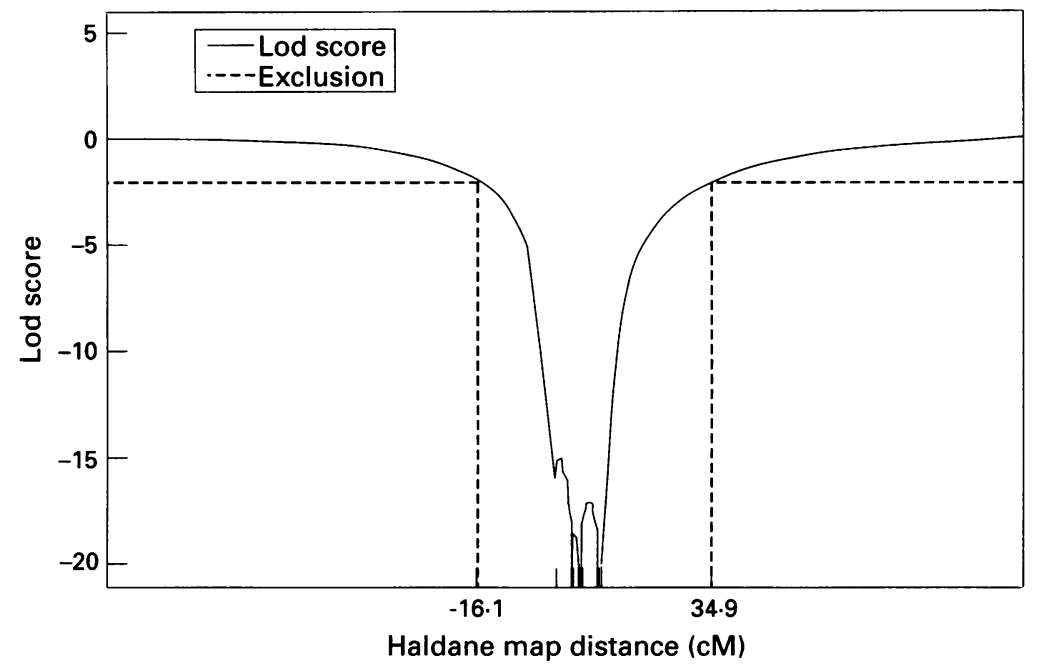

Figure 2 Exclusion map with no heterogeneity: DCM affecteds only multipoint lod scores for chromosome 6 markers with genetic map: tel-299-(4cM)-276-(0.6 cM)$105(0 \cdot 4 \mathrm{cM})-273-(4 \mathrm{cM})-291-c e n$. The solid line is the multipoint lod score and the broken line indicates the region of exclusion where the lod score is less than -2 .

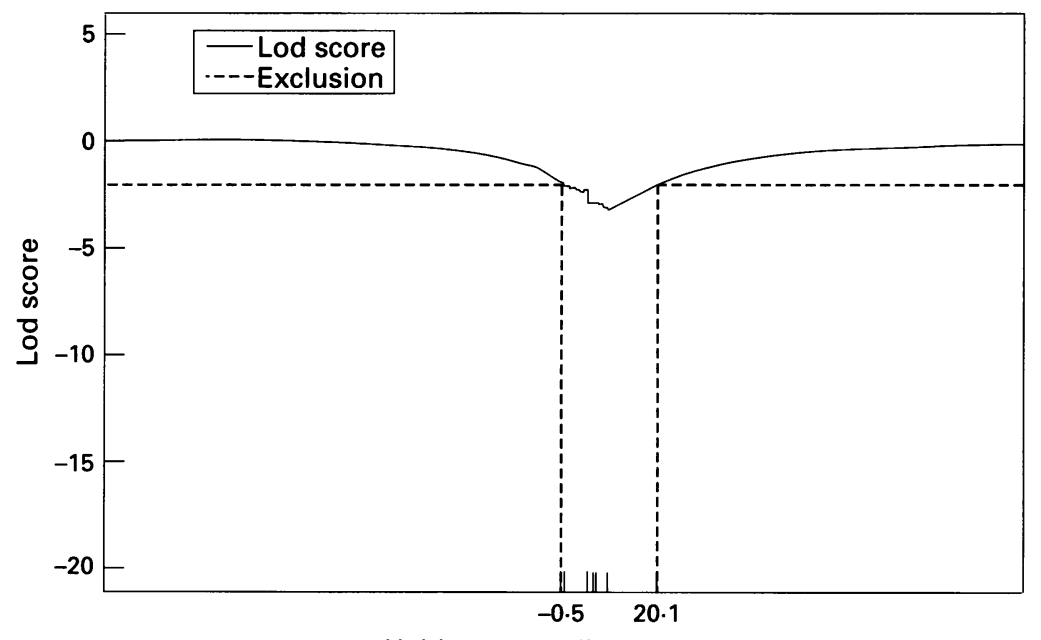

Haldane map distance (cM)

Figure 3 Exclusion map with 60\% of DCM attributable to HLA: DCM affecteds multipoint lod scores for chromosome 6 markers with genetic map: tel-299-(4 cM)-276$(0.6 c M)-105(0.4 c M)-273-(4 c M)-291-c e n$. The solid line is the multipoint lod score and the broken line indicates the region of exclusion where the lod score is less than -2.

region. We found similar results when we allowed the genetic distance between D6S276 and D6S273 to increase from $1 \mathrm{cM}$ to as great as $5 \cdot 1 \mathrm{cM}$, as suggested by Martin et al. ${ }^{23}$

\section{Discussion}

Case-control studies have established an association between DCM and certain HLA class II antigens, particularly DR4 and DQw4. ${ }^{1}$ These studies imply that the pathogenesis of DCM could be attributed to genetically regulated immune response factors in a subset of cases. Recently, exclusion of linkage to the HLA locus in one family of moderate size was reported. ${ }^{10}$ Because of possible genetic heterogeneity, study of additional families with DCM for linkage to the HLA region was considered to be of interest. The purpose of our study was to test the hypothesis that patients with DCM in our 12 pedigrees may have a primary gene defect within the HLA region. Because DCM is associated with high mortality, and individual pedigrees were of relatively small size, the families identified had relatively few living affected members. Consequently, linkage to the HLA region could not be independently excluded for every family using the convention of a lod score $<-2$. Only four pedigrees had a lod score $<-2$ at a recombination fraction of zero for at least one of the markers. Linkage analysis was performed using five polymorphic markers spanning $9 \mathrm{cM}$ and including the HLA locus on chromosome $6 \mathrm{p} 21.3$. However, the HLA locus could be excluded based on the total lod score for all 12 pedigrees using two point analyses; a minimum distance of $16 \mathrm{cM}$ could be excluded using multipoint analyses with all families potentially linked, and a minimum distance of $0.5 \mathrm{cM}$ excluded when no less than $60 \%$ of DCM is attributable to linkage with that region. We recognise that the power of such analyses is limited by the relatively small number of pedigrees and the small sizes and limited informativeness of the pedigrees. Although it is possible that a genetically distinct subset of these families could be linked to the HLA region, this subset is probably less than $60 \%$ of our DCM families. Lod scores were negative for nine of $12 \mathrm{DCM}$ families at recombination frequencies of $0-20 \%$ for D6S273, the marker that is most closely linked to the HLA class II DR locus.

This study provides additional support for the conclusions by Krajinovic et $a l^{10}$ and Carlquist $e t a l^{8}$ that DCM cannot be explained solely by the presence or absence of a single HLA allele, by providing evidence for exclusion of a primary gene defect at the HLA locus in a series of families with DCM. Also in agreement with their conclusion, this does not exclude the possibility that HLA regulated immune response factors could have a modifying effect on disease penetrance and expression. This study provides evidence against an unidentified gene in the HLA locus region as the primary determinant of DCM in our families, and thus provides support for the possible modifying effects of HLA genes on the disease.

The authors gratefully acknowledge partial support for this work from the John Patrick Albright Foundation and National Institutes of Health institutional training grant HL07111 to TMO.

1 Carlquist JF, Menlove RL, Murray MB, O'Connell JB, Anderson JL. HLA class II (DR and DQ) antigen associations in idiopathic dilated cardiomyopathy: validation study and meta-analysis of published HLA association studies. Circulation 1991;83:515-22.

2 Limas C, Limas CJ, Boudoulas H, et al. T-cell receptor gene polymorphisms in familial cardiomyopathy: correlation with anti- $\beta$-receptor autoantibodies. Am Heart $f$ 1992;124:1258-63.

3 Fallon JT. Myocarditis and dilated cardiomyopathy: different stages of the same disease? In: Waller BF, ed. Cardiovascular clinics: contemporary issues in cardiovascula pathology. Philadelphia, FA Davis, 1988:155-62.

4 Mercadier J, Hatem S, Schwartz K. Dilated cardiomyopathies: molecular changes responsible for altered contraction and relaxation. Heart Failure 1993;9:112-20.

5 Lange LG, Schreiner GF. Immune mechanisms of cardiac disease. N Engl f Med 1994;330:1129-35.

6 Bender JR. Idiopathic dilated cardiomyopathy: an immunologic, genetic, or infectious disease, or all of the above? Circulation 1991;83:704-6.

7 Yunis EJ, Yunis I. Update of the human major histocompatability complex. Transplantation Proc 1991;23: 1734-7.

8 Carlquist JF, Ward RH, Husebye D, Feolo M, Anderson JL. Major histocompatibility complex class III gene frequencies by serologic and deoxyribonucleic acid genomic 
typing in idiopathic dilated cardiomyopathy. $A m \mathcal{F}$ Cardiol 1994;74:918-20.

9 Koike S, Kawa S, Yabu K, et al. Familial dilated cardiomyopathy and human leukocyte antigen: a report of two family cases. $\mathcal{F p}_{p}$ Heart $\mathcal{f} 1987 ; 28: 941-5$.

10 Krajinovic M, Mestroni L, Severini GM, et al. Absence of linkage between idiopathic dilated cardiomyopathy and candidate genes involved in the immune function in large Italian pedigree. $\mathcal{F}$ Med Genet 1994;31:766-71.

11 Michels VV, Moll PP, Miller FA, et al. The frequency of familial dilated cardiomyopathy in a series of patients with idiopathic dilated cardiomyopathy. N Engl f Med 1992; 326:77-82.

12 Goerss JB, Michels VV, Burnett J, et al. Frequency of familial dilated cardiomyopathy. Eur Heart f (in press).

13 Roder JC, Cole SP, Kozbor D. The EBV-hybridoma technique. Methods Enzymol 1986;121:140-67.

14 Weber JI May PE. Abundant class of human DNA polymorphisms which can be typed using the polymerase chain reaction. Am $₹$ Hum Genet 1989;44:388-96.

15 NIH/CEPH Collaborative Mapping Group. A comprehensive genetic linkage map of the human genome. prehensive genetic linka
Science 1992;258:67-86.
16 Gyapay G, Morissette J, Vignal A, et al. The 1993-94 Genethon human genetic linkage map. Nature Genet 1994; 7:246-339.

17 Volz A, Boyle JM, Cann HM, Cottingham RW, Orr HT, Ziegler A. Report of the second international workshop on human chromosome 6. Genomics 1994;21:464-72.

18 Weber JL, Kwitek AE, May PE, Zoghbi HY. Dinucleotide repeat polymorphism at the D6S105 locus. Nucleic Acids Res 1991;19:968.

19 Lathrop GM, Lalouel JM. Easy calculation of lod scores and genetic risks on small computers. Am $\mathcal{f}$ Hum Genet 1984;36:360-5.

20 Lathrop GM, Lalouel JM, Julier C, Ott J. Multilocus linkage analysis in humans: detection of linkage and estimation of recombination. Am f Hum Genet 1985;37:482-98.

21 Risch N, Giuffra L. Model misspecification and multipoint linkage analysis. Hum Hered 1992;42:77-92.

22 Ott J. Linkage analysis and family classification under heterogeneity. Ann Hum Genet 1983;47:311-20.

23 Martin M, Mann D, Carrington M. Recombination rates across the HLA complex; use of microsatellites as a rapid screen for recombinant chromosomes. Hum Mol Genet screen for recom 